\title{
Human zona pellucida recognition associated with removal of sialic acid from human sperm surface
}

\author{
B. Lassalle and J. Testart \\ INSERM unité 355,32 rue des Carnets 92140 Clamart, France
}

\begin{abstract}
The ability of human spermatozoa recovered from highly motile sperm fractions to bind wheat germ agglutinin (WGA) after discontinuous Percoll gradient centrifugation was studied. WGA could bind to almost all motile spermatozoa, whereas fewer than $25 \%$ of spermatozoa could bind peanut (PNA) and concanavalin A (Con A) agglutinin, two lectins that specifically bind acrosomal membranes. After removal of the plasma membrane with $0.04 \%$ Triton X100, WGA, PNA and Con A bound more than $80 \%$ of spermatozoa, but binding sites for WGA on the anterior acrosomal region were markedly reduced. The expression of sialic acid on human sperm plasma membrane was demonstrated, since WGA, which specifically recognizes both sialic acid (NeuNAc) and $N$-acetylglucosamine (GlcNAc), bound almost all intact motile spermatozoa, whereas succinylated WGA, which recognizes only GIcNAc, bound less than 10\% of intact motile spermatozoa. Moreover, binding of WGA was compared with that of three other lectins (Sambucus nigra, SNA; Maackia amurensis, MAL and Limulus polyphemus, LPA) with specificity for different NeuNAc linkages. Only SNA, which requires the presence of the disaccharide structure NeuNAc $\alpha(2,6) \mathrm{Gal} / \mathrm{GalNAc}$, showed a positive correlation with sperm motility as observed with WGA. Moreover, there was a strong inhibition of WGA binding on spermatozoa preincubated with bovine submaxillary mucin containing $(2,6)$-linked NeuNAc. These results demonstrate the presence of NeuNAc $\alpha(2,6) \mathrm{Gal} / \mathrm{GalNAc}$ glycoconjugate sequences on the plasma membrane of the motile human spermatozoon. Treatment of spermatozoa with Arthrobacter ureafaciens neuraminidase to cleave NeuNAc residues led to a dose-dependent decrease of WGA binding at the sperm surface and to the enhancement of sperm attachment to the zona pellucida. We hypothesize that the release of sialic acid from the sperm plasma membrane could be one of the capacitation events necessary for unmasking certain sperm surface antigens implicated in zona pellucida recognition.
\end{abstract}

\section{Introduction}

Maturation of the spermatozoon (acquisition of motility and fertilizing ability) seems to be acquired through the epididymis (Orgebin-Crist and Fournier-Delpech, 1982; Bellvé and O'Brien, 1983; Primakoff et al., 1987). Many modifications of the sperm surface, such as masking or unmasking surface molecules or addition of new molecules, result from interactions between the spermatozoa and the surrounding epididymal fluid and especially with glycoproteins synthesized and secreted by the epididymal epithelium (Brown et al., 1983; Eddy et al., 1985; Srivastava and Olson, 1991).

Sialic acid (NeuNAc) residues are secreted by the epithelium of the epididymis as terminal sugars of sialoglycoproteins that bind to the sperm surface during epididymal transit (reviewed by Toshimori et al., 1991). Sialic acid residues have significant biological functions such as masking of antigens, i.e. anti-

Address reprint requests to Bruno Lassalle, INSERM unité 355,32 rue des Carnets 92140 Clamart, France.

Received 24 January 1994. recognition molecules (reviewed by Schauer, 1985) or as receptor determinants (Herrler et al., 1992; Yoshida et al., 1992; Powell et al., 1993). The presence of sialic acid bound to the surface of epididymal spermatozoa has been demonstrated in several mammals (rats: Toowicharanont and Chulavatnatol, 1983; mice: Toshimori et al., 1991; rams and bulls: Holt, 1980). Sperm surface sialic acid seems to be conserved, at least in rams and bulls, after ejaculation (Holt, 1980). The loss of sperm surface sialic acid allows phagocytosis of mouse spermatozoa by macrophages in vitro (Toshimori et al., 1991). Seminal phagocytic cells play a role in the destruction of abnormal human spermatozoa from the ejaculate (Tomlinson et al., 1992) and leucocytes are recruited at the human cervix in a specific response to spermatozoa (Pandya and Cohen, 1985). The presence of sperm surface sialic acid may render certain spermatozoa immune from phagocytosis (Holt, 1980), whereas its absence leads to the destruction of spermatozoa of abnormal morphology by phagocytosis in the ejaculate and during their transit through the female genital tract.

The aim of our work was to study (i) the presence of sialic acid at the sperm surface either on the plasma membrane before 
Table 1. Lectins used in this study and their sugar specificities

\begin{tabular}{|c|c|c|}
\hline Lectins & Sugar specificities & References \\
\hline $\begin{array}{l}\text { PNA } \\
\text { Peanut agglutinin } \\
\text { Arachis hypogaea } \\
\text { (Sigma) }\end{array}$ & D-Gal $\beta$ 1,3 D GalNAc $>$ D GalNAc $>$ D Gal & 1 \\
\hline $\begin{array}{l}\text { Con A } \\
\text { Concanavalin A } \\
\text { Canavalia ensiformis } \\
\text { (Sigma) }\end{array}$ & $\begin{array}{l}\text { Man } \alpha-1,2 \text { Man } \alpha-1,2 \text { Man }>\text { Man } \alpha-1,2 \\
\operatorname{Man}>\alpha \text { Glc }>\alpha \text { GlcNAc }\end{array}$ & 2 \\
\hline $\begin{array}{l}\text { Succinylated WGA } \\
\text { Wheat germ agglutinin } \\
\text { Triticum vulgaris } \\
\text { (Vector) }\end{array}$ & $\begin{array}{l}\text { GlcNAc } \beta-1,4 \text { GlcNAc } \beta-1,4 \text { GlcNAc }>\text { GlcNAc } \beta-1,4 \\
\text { GlcNAc }>\text { GlcNAc }\end{array}$ & 2 \\
\hline $\begin{array}{l}\text { WGA } \\
\text { Wheat germ agglutinin } \\
\text { Triticum vulgaris } \\
\text { (Sigma) }\end{array}$ & $\begin{array}{l}\text { NeuNAc } \alpha-2,6 \text { and } \alpha-2,3 \text { D Gal/GalNAc and } \\
\text { GlcNAc } \beta-1,4 \text { GlcNAc }\end{array}$ & $\begin{array}{l}3-5 \\
2,4\end{array}$ \\
\hline $\begin{array}{l}\text { MAL } \\
\text { Maackia amurensis } \\
\text { leukoagglutinin } \\
\text { (Vector) }\end{array}$ & $\begin{array}{l}\text { Necessitate the trisaccharide structure: } \\
\text { NeuNAc } \alpha-2,3 \text { Gal } \beta-1,4 \text { GlcNAc/Glc }\end{array}$ & $6-8$ \\
\hline $\begin{array}{l}\text { SNA } \\
\text { Elderberry bark agglutinin } \\
\text { Sambucus nigra } \\
\text { (Vector) }\end{array}$ & $\begin{array}{l}\text { Necessitate the disaccharide structure: } \\
\text { NeuNAc } \alpha-2,6 \mathrm{Gal} / \mathrm{GalNAc}\end{array}$ & $6,8,9$ \\
\hline $\begin{array}{l}\text { LPA } \\
\text { Horseshoe crab agglutinin } \\
\text { Limulus polyphemus } \\
\text { (Sigma) }\end{array}$ & $\begin{array}{l}\text { NeuNAc } \alpha-2,3 \text { GalNAc }>\text { NeuNAc } \alpha-2,6 \\
\text { GalNAc }>\text { NeuNAc } \\
\text { GalNAc }\end{array}$ & 10 \\
\hline
\end{tabular}

and after capacitation or after the loss of the plasma membrane and (ii) the relationships between sialic acid expression and sperm fertilizing ability of spermatozoa.

\section{Materials and Methods}

\section{Preparation of spermatozoa}

Ejaculated fresh semen was obtained with consent from normal donors and liquefied for $30 \mathrm{~min}$ at $37^{\circ} \mathrm{C}$. Whole semen, $500 \mu \mathrm{l}$, was diluted with $5 \mathrm{ml}$ PBS plus $0.4 \%(\mathrm{w} / \mathrm{v})$ BSA (fraction V, Sigma La Verpillère), and then centrifuged at $600 \mathrm{~g}$ for $7 \mathrm{~min}$. The sperm pellet was resuspended in $1 \mathrm{ml} \mathrm{B2}$ medium (B2 Menezo, Biomerieux SA, 69280 Marcy-l'Etoile). This sample constituted the entire sperm sample. One millilitre of whole semen was separated on a discontinuous Percoll gradient as described by Lassalle and Testart (1992) except that two concentrations (90 and $45 \%$ ) of Percoll were used rather than three. After centrifugation $(25 \mathrm{~min}$ at $600 \mathrm{~g}$ ), Percoll fractions were carefully separated and washed by dilution with $5 \mathrm{ml} \mathrm{PBS}-\mathrm{BSA}$ and then centrifuged at $600 \mathrm{~g}$ for $7 \mathrm{~min}$. The pellets were resuspended in $1 \mathrm{ml} \mathrm{B2}$ medium. Before addition of lectin probes, the numbers of motile and immotile spermatozoa were determined on a Malassez cell to estimate the percentage of motile spermatozoa for each sperm suspension.

\section{Labelling of spermatozoa with lectins}

The ability of spermatozoa from selected populations to bind particular lectins conjugated to fluorescein isothiocyanate (FITC) or biotin was studied. Lectins were purchased from Sigma or Vector (Vector Laboratories Inc., Burlingame, CA). The common names, taxonomic names and specificities of these lectins are given (Table 1 ). Wheat germ agglutinin (WGA) and succinylated WGA (sWGA) were used to demonstrate the presence of cell surface receptors containing sialic acid (Monsigny et al., 1980). WGA can recognize both NeuNAc (sialic acid) and GlcNAc ( $N$-acetylglucosamine), whereas sWGA recognizes only GlcNAc (Monsigny et al., 1980). Peanut agglutinin (PNA) and concanavalin A (Con A) were chosen for their ability to bind specifically to the outer and the inner acrosomal membrane of human spermatozoa, respectively 
(Mortimer et al., 1987; Holden et al., 1990), and were used to assess sperm acrosomal status when compared with WGA. WGA was also compared to Limulus polyphemus (LPA), Maackia amurensis (MAL) and Sambucus nigra (SNA) agglutinins that exhibit specificity for different NeuNAc-linkage from Gal/GalNAc (Table 1).

Each treated sperm suspension was incubated for $30 \mathrm{~min}$ at $37^{\circ} \mathrm{C}$ with lectin at optimal concentrations according to previous reports (Table $\mathrm{I}$ ) i.e. $10 \mu \mathrm{g} \mathrm{ml}^{-1}$ (PNA, Con A) or $5 \mu \mathrm{g}$ $\mathrm{ml}^{-1}$ (SNA, MAL, LPA, WGA and sWGA). Lectin-treated sperm suspensions were washed twice in PBS-BSA and pellets were resuspended in $100 \mu \mathrm{l}$ PBS-BSA. Sperm pellets from samples pretreated with biotinylated lectins were incubated for an additional $30 \mathrm{~min}$ at $37^{\circ} \mathrm{C}$ with $10 \mu \mathrm{l}$ of streptavidin-FITC $\left(0.25 \mathrm{mg} \mathrm{ml}^{-1}\right)$ solution (Sigma) and then washed twice in PBS-BSA.

Changes in lectin binding after incubation of spermatozoa in vitro and release of sperm plasma membrane

Binding of PNA, Con A, WGA and sWGA to the sperm head was also studied after incubation of spermatozoa in vitro or treatment that induces total release of the sperm plasma membrane. Incubation of spermatozoa in vitro was performed at $4^{\circ} \mathrm{C}$ for $24 \mathrm{~h}$. Total release of the sperm plasma membrane was induced by treatment with $0.04 \%$ Triton $X_{100}$ for $30 \mathrm{~min}$ at $37^{\circ} \mathrm{C}$ (Langlais et al., 1981; Giroux-Widemann et al., 1991). Ultrastructural studies have found that about one-third of fresh (Barthelemy et al., 1990) and all Triton X100-treated (Langlais et al., 1981; Giroux-Widemann et al., 1991) human spermatozoa have lost their plasma membrane. Sperm samples that had been incubated or treated with Triton X100 were diluted with $5 \mathrm{ml}$ PBS-BSA before centrifugation $(600 \mathrm{~g}$ for $10 \mathrm{~min})$ and finally the pellet was resuspended in $1 \mathrm{ml} \mathrm{B} 2$ medium before addition of lectin probes.

\section{Microscope analysis of lectin binding}

Sperm suspensions, $10 \mu \mathrm{l}$, were placed between slide and coverslip for observation under a Leitz microscope equipped with epifluorescence. Percentages of spermatozoa displaying fluorescence anywhere on the head surface (i.e. whatever the precise binding location on the sperm head) or on the anterior acrosomal region were estimated from at least 100 spermatozoa per preparation. Binding of PNA, Con A, WGA and sWGA to the anterior acrosomal region was studied for untreated spermatozoa recovered from the $90 \%$ Percoll fractions and after treatment with Triton $X_{100}$. WGA binding on the anterior acrosomal region of untreated spermatozoa was compared with that of capacitated spermatozoa and of those recovered in the $45 \%$ Percoll fraction.

\section{Inhibition of sperm WGA binding by glycoproteins or polysaccharides containing terminal NeuNAc GallGaN $A c$ sequences}

Sugar-binding specificity of lectins is usually determined by measuring the ability of a sugar to inhibit either blood cell agglutination by the lectin, precipitation of the lectin by polysaccharides or glycoproteins or binding of the lectin to glycosubstances (reviewed by Zeng and Gabius, 1992). WGAFITC, $5 \mu \mathrm{g} \mathrm{ml}^{-1}$, in $1 \mathrm{ml} \mathrm{B2}$ medium was incubated alone (control) or with increasing concentrations of either $\mathrm{N}$-acetylneuraminic acid (Sigma), bovine submaxillary mucin, BSM (Sigma) or bovine fetuin (Sigma) to compare the effects of glycoproteins containing terminal NeuNAc linked $\alpha 2,3, \alpha 2,6$ or both with penultimate Gal or GalNAc. After incubation for $1 \mathrm{~h}$ at $37^{\circ} \mathrm{C}, 10^{\circ}$ spermatozoa $(100 \mu \mathrm{l})$ from the highly motile sperm fraction ( $90 \%$ Percoll fraction) were added. These mixtures were incubated for an additional $1 \mathrm{~h}$ at $37^{\circ} \mathrm{C}$ and spermatozoa were then washed twice before observation under a microscope. The percentage of spermatozoa showing fluorescence on the head surface was estimated for each inhibitor tested. Concentrations of fetuin and BSM were expressed on the basis of NeuNAc content. Results were given as total inhibition (absence of WGA bound spermatozoa) or $50 \%$ inhibition when percentages of spermatozoa with bound WGA reached $50 \%$ of the control value.

\section{Effect of sialic acid release by neuraminidase on sperm-zona pellucida recognition}

A preliminary experiment was done to study WGA binding on the sperm head after treatment with increasing concentrations of neuraminidase from Arthrobacter ureafaciens (Sigma). Spermatozoa recovered from the $90 \%$ Percoll fraction were treated for $1 \mathrm{~h}$ with $0,200,300$ or $500 \mathrm{mU}$ neuraminidase $\mathrm{ml}^{-1}$ at $37^{\circ} \mathrm{C}$, and washed in PBS-BSA. Sperm pellets were then resuspended in I ml B2. Labelling with WGA-FITC, washing and control of WGA binding were performed as described above.

Human oocytes that were not fertilized by $40-48 \mathrm{~h}$ after insemination in vitro were stored at $4^{\circ} \mathrm{C}$ in ammonium sulfate solution for optimal conservation of the zona pellucida (Yoshimatsu et al., 1988). Two hours before insemination, the zonae pellucidae were thoroughly rinsed for $90 \mathrm{~min}$ in four successive baths of PBS-BSA and then placed for $30 \mathrm{~min}$ in B2 medium at $37^{\circ} \mathrm{C}$ before insemination. Spermatozoa recovered from the $90 \%$ Percoll fraction were either untreated or treated for $\mathrm{I} \mathrm{h}$ with $500 \mathrm{mU}$ neuraminidase $\mathrm{ml}^{-1}$ at $37^{\circ} \mathrm{C}$ before washing in PBS-BSA. Sperm pellets were labelled by suspen-

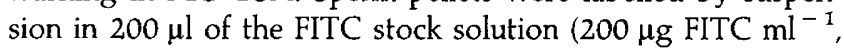
Sigma) according to Liu et al. (1988). The suspension was incubated at $37^{\circ} \mathrm{C}$ for $15 \mathrm{~min}$ and spermatozoa were washed twice by centrifugation $(600 \mathrm{~g}$ for $7 \mathrm{~min})$, resuspended in $\mathrm{B} 2$ medium and diluted to a concentration of $5 \times 10^{5}$ spermatozoa $\mathrm{ml}^{-1}$ with B2 medium. Five hundred microlitres of untreated or neuraminidase-treated sperm suspensions were placed in macrowells of Nunclon delta dishes (Nunc, Roskilde) before addition of zonae pellucidae. After incubation for $2 \mathrm{~h}$ (at $37^{\circ} \mathrm{C}$ under $5 \% \mathrm{CO}_{2}$ ), zonae pellucidae were thoroughly rinsed in three successive baths of PBS-BSA and then placed between slide and coverslip as described by Lassalle and Testart (1991). Observations were made under a Leitz microscope equipped with epifluorescence and the number of fluorescent spermatozoa on each zona pellucida was determined. These experiments to evaluate the ability of human spermatozoa to bind human zonae pellucidae were approved by the French Comité National d'Ethique. 


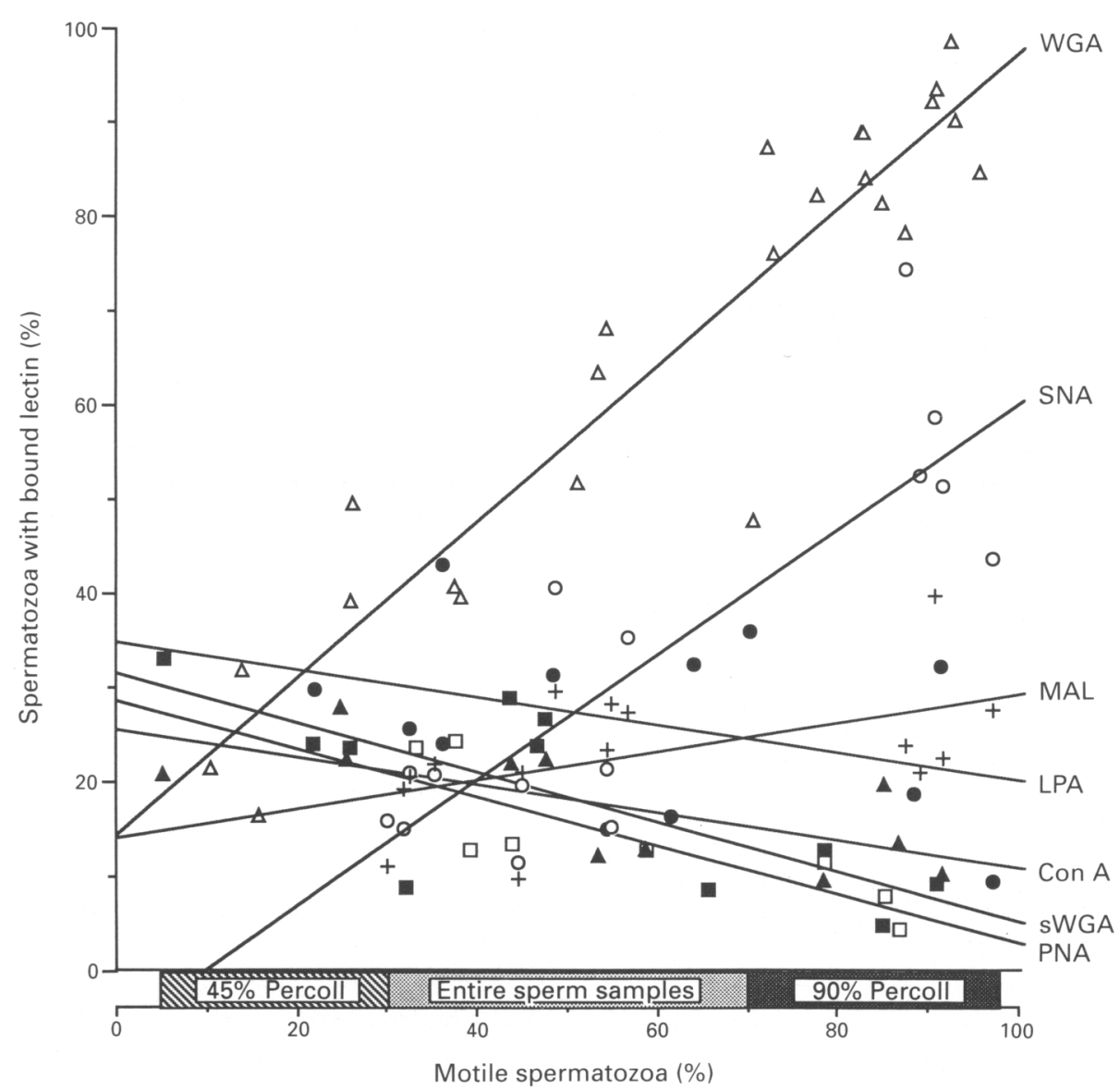

Fig. 1. Lectin binding according to sperm motility. Entire sperm samples and sperm populations recovered in the $45 \%$ and $90 \%$ Percoll fractions after Percoll density gradient centrifugation were obtained from each ejaculate. Percentages of motile spermatozoa were $34.0-56.9 \%, 70.4-95.5 \%$ and $5.2-30.0 \%$ in the entire sperm samples, and in the $90 \%$ and $45 \%$ Percoll fractions, respectively. Equations of the regression straight line and correlation coefficients were obtained and linearity was tested using $F$ tests. PNA: $R^{2}=0.70, P<0.01$; Con $A, R^{2}=0.50, P<0.02$; sWGA, $R^{2}=0.56$, $P<0.01$; WGA, $R^{2}=0.90, P<0.0001$; SNA, $R^{2}=0.72, P<0.001 ; \mathrm{MAL}, R^{2}=0.27, P<0.05$. No significant correlation was found between LPA binding and sperm motility. At least four ejaculates from different normal donors were used for each lectin tested. ( $\square$ ) PNA, peanut agglutinin; ( $\boldsymbol{\Delta}$ ) Con $A$, concanavalin $A ;(\triangle)$ WGA, wheat germ agglutinin; (D) sWGA, succinylated wheat germ agglutinin; (-) LPA, Limulus polyphemus agglutinin; (+) MAL, Maackia amurensis leukoagglutinin and (O) SNA, Sambucus nigra agglutinin.

\section{Statistical analysis}

Data were analysed using Statview 4.0 software provided by a Macintoch II si computer. Means ( \pm SEM) were compared using Student's $t$ test and one-way analysis of variance (ANOVA). Regression equations and correlation coefficients were obtained and linearity was tested using $F$ tests.

\section{Results}

Labelling of spermatozoa with lectins according to sperm motility

Percentages of motile spermatozoa were $34.0-56.9 \%$, $70.4-95.5 \%$ and $5.2-30.0 \%$ in the entire sperm sample, in the $90 \%$ and in the $45 \%$ Percoll fractions, respectively. For three of the tested lectins, PNA, Con A and sWGA, percent- ages of spermatozoa that exhibited positive lectin binding on the sperm head decreased (PNA, $R^{2}=0.70, P<0.01$; Con $\mathrm{A}$, $R^{2}=0.50, P<0.02 ;$ sWGA, $R^{2}=0.56, P<0.01$ ) in relation to the increasing proportion of motile spermatozoa (Fig. 1) and varied between 14 and $25 \%$. On the contrary, WGA bound $76 \%$ or $33 \%$ of spermatozoa when sperm motility was higher or lower than $50 \%$, respectively, and a positive correlation $\left(R^{2}=0.90, P<0.0001\right)$ was observed between WGA binding and the number of motile spermatozoa (Fig. 1). Binding of WGA occurred in nearly all the motile spermatozoa, especially those from the $90 \%$ Percoll fraction (Fig. 1). A positive correlation was also observed between motility and lectin binding for SNA $\left(R^{2}=0.72, P<0.001\right)$ and MAL $\left(R^{2}=0.27, P<0.05\right)$ (Fig. 1). However, when the sperm motility was higher than $50 \%$, SNA and MAL bound $44 \%$ and $27 \%$ of spermatozoa, respectively (data not 


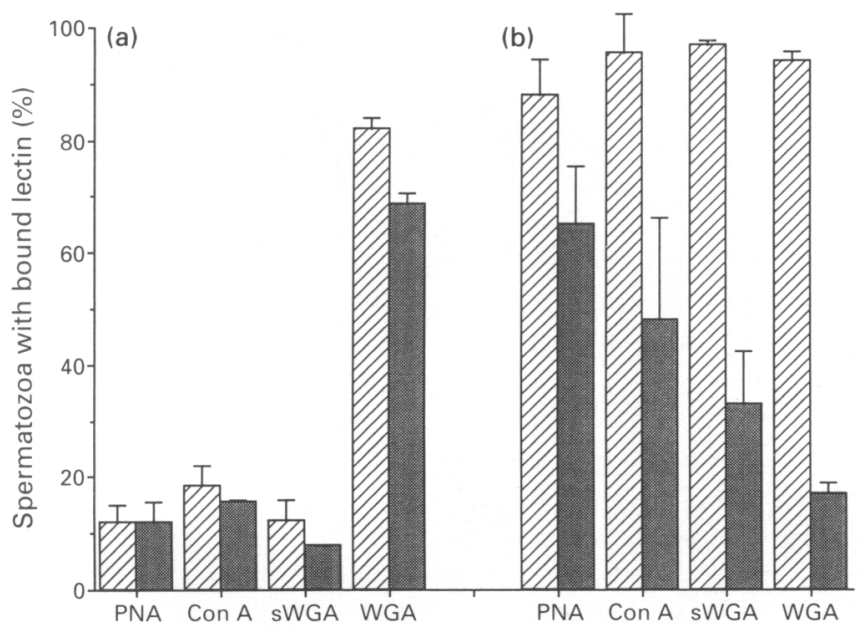

Fig. 2. Binding sites of lectins on the head of spermatozoa (a) untreated and (b) treated with Triton X100. Wheat germ agglutinin (WGA) was compared with peanut agglutinin (PNA), concanavalin A (Con A) and succinylated wheat germ agglutinin (sWGA) lectins. ( $\triangle$ ) lectin-binding sites on sperm heads whatever the precise location; lectin-binding sites at the anterior acrosomal region. Data are means \pm SEM from at least three experiments.

shown), compared with $76 \%$ using WGA. No significant correlation was found between LPA binding and sperm motility (Fig. 1).

Lectin distribution in relation to incubation of spermatozoa in vitro and release of sperm plasma membrane

Proportions of untreated or Triton X100-treated spermatozoa with binding sites for PNA, Con A, sWGA and WGA on the sperm head or at least on the anterior acrosomal region were compared (Fig. 2). Fewer than $20 \%$ of untreated spermatozoa bound PNA, Con A and sWGA, whereas WGA binding occurred in at least $80 \%$ of this sperm population (Fig. 2). After sperm demembranation with Triton X100, all tested lectins bound more than $80 \%$ of spermatozoa (Fig. 2). Whereas proportions of demembranated spermatozoa with lectin binding sites on the anterior acrosomal region tended to increase for PNA (12.0 versus $65.1 \%, P<0.01$ ), Con $A$ (15.6 versus $48.1 \%$, not significant) and sWGA (7.7 versus $33.0 \%$, not significant), these proportions decreased for WGA $(68.9 \%$ versus $16.9 \%, P<0.001$ ) (Figs 2 and 3 ). WGA bound particularly on the equatorial segment and the post-acrosomal region (with or without binding on the anterior acrosomal region) after Triton $\mathrm{X} 100$ treatment but similar proportions of untreated $(76.2 \%)$ and Triton $X_{100}$-treated $(73.6 \%)$ spermatozoa had binding sites for these two sperm regions (data not shown).

Preliminary studies in which incubation was for $24 \mathrm{~h}$ at $4^{\circ} \mathrm{C}$ showed that percentages of acrosome-reacted spermatozoa assessed by PNA-FITC or Con A-FITC, two lectins used to assess sperm acrosome reaction (Mortimer et al., 1987; Holden et al., 1990), were higher $(P<0.05)$ for capacited $(39.3 \%)$ than for untreated spermatozoa (15.1\%) (data not shown). Proportions of spermatozoa that bound WGA anywhere on the sperm head surface or on the anterior acrosome were lower in the
45\% Percoll fraction and after incubation compared with that in spermatozoa from the $90 \%$ Percoll fraction (Fig. 3). However, after sperm demembranation with Triton X100, only proportions of those spermatozoa that exhibit WGA binding on the anterior acrosomal region were low (Fig. 4).

Inhibition of sperm WGA binding by glycoproteins or polysaccharides containing terminal NeuNAc Gal/GalNAc sequences

Total inhibition of WGA binding on the sperm head was observed for $4 \mathrm{mmol}$ NeuNAc $1^{-1}$ contained in bovine submaxillary mucin (Fig. 5). About $10 \mathrm{mmol} \mathrm{NeuNAc} \mathrm{l}^{-1}$ contained in fetuin or $150 \mathrm{mmol}$ free NeuNAc ${ }^{-1}$ were needed for $50 \%$ inhibition of WGA binding and total inhibition was not reached with $20 \mathrm{mmol}$ fetuin $1^{-1}$ (Fig. 4). No sign of WGA inhibition was observed with $32 \mathrm{mmol}$ NeuNAc $\mathrm{l}^{-1}$ contained in NeuNAc $\alpha(2,3)$ lactose and $50 \%$ inhibition was obtained with

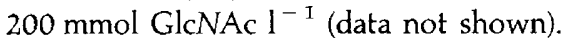

Effects of treatment of spermatozoa with neuraminidase on binding of WGA to spermatozoa and sperm-zona pellucida recognition

Neuraminidase from Arthrobacter ureafaciens has been used to cleave $\alpha(2,3), \alpha(2,6)$ or $\alpha(2,8)$-linked NeuNAc residues (Powell et al., 1993). Binding sites for WGA were observed on 73.4, $39.6,31.4$ and $20.2 \%$ of spermatozoa treated with $0,200,300$ and $500 \mathrm{mU}$ Arthrobacter ureafaciens neuraminidase $\mathrm{ml}^{-1}$, respectively (Fig. 6). Neuraminidase treatment induced a dosedependent decrease $\left(R^{2}=0.87, P<0.01\right)$ in the percentage of spermatozoa that bound WGA. Treatment of spermatozoa with $500 \mathrm{mU}$ Arthrobacter ureafaciens neuraminidase $\mathrm{ml}^{-1}$ did not affect the sperm motility and the number of motile spermatozoa that were identical to untreated sperm samples. Moreover, no sign of sperm agglutination or adhesion to the plastic culture dish was observed after neuraminidase treatment. In three assays using different sperm donors, the average number of spermatozoa bound per human zona pellucida increased significantly (donor $1,2.9$ versus 18.0, $P<0.001$; donor 2, 3.4 versus 15.8, $P<0.001$; donor $3,16.9$ versus 35.4 , $P<0.01$ ) after pretreatment of spermatozoa with $500 \mathrm{mU}$ Arthobacter ureafaciens neuraminidase $\mathrm{l}^{-1}$ (Table 2, Fig. 7).

\section{Discussion}

Human spermatozoa are characterized by a high heterogeneity in morphology, motility and nuclear maturity. Percoll density gradient centrifugation is used to separate sperm populations according to sperm density (Bolton and Braude, 1984) and spermatozoa with a dense and homogeneous nucleus were recovered mainly in the highest density fraction $(90 \%)$ of the Percoll gradient. Spermatozoa of normal and motile spermatozoa are mainly recovered in the $90 \%$ Percoll fraction (Le Lannou and Blanchard, 1988) and that fraction contains spermatozoa with high fertilizing ability (Hyne et al., 1983; Berger et al., 1985; Sapienza et al., 1993). Our results show that less than $20 \%$ of freshly ejaculated spermatozoa recovered in the $90 \%$ Percoll fraction exhibited positive PNA, Con A and 

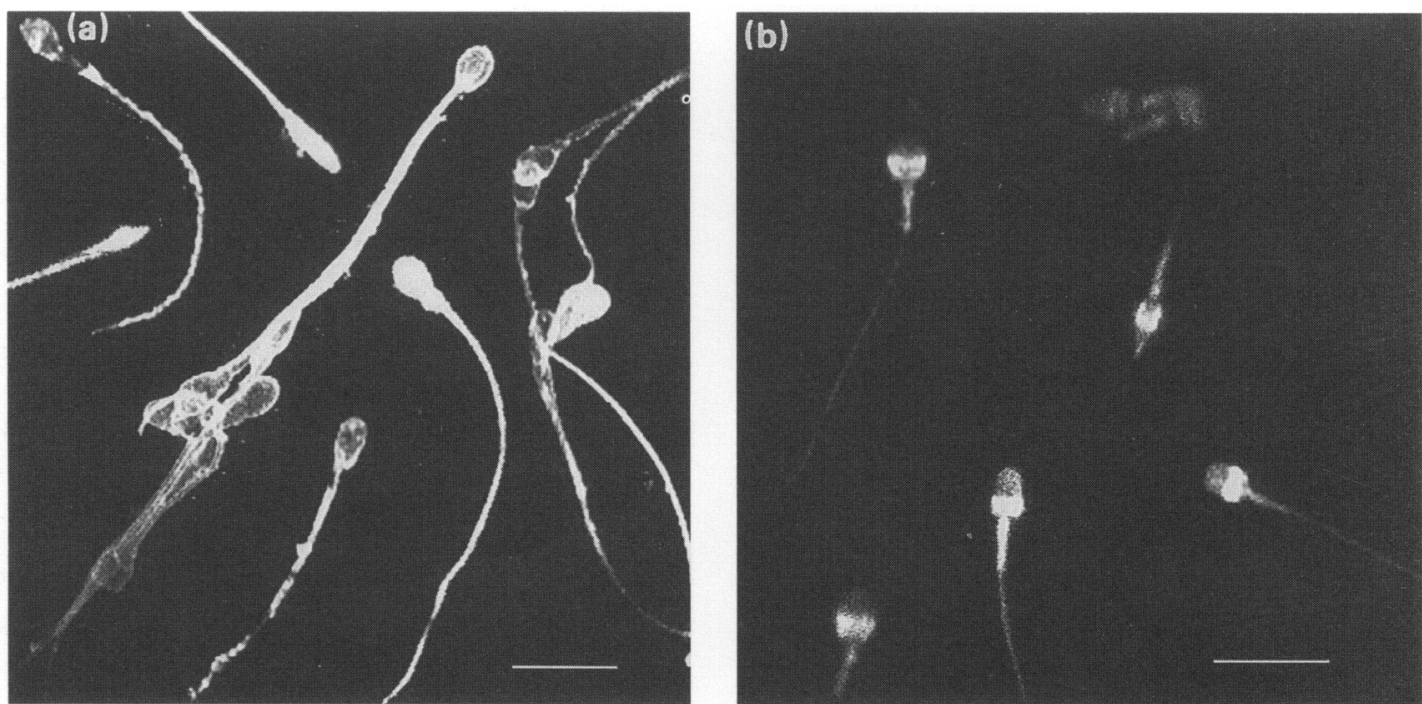

Fig. 3. (a) Untreated spermatozoa from the $90 \%$ Percoll fraction exhibited wheat germ agglutinin (WGA)-fluorescein isothiocyanate (FITC) binding on all surfaces including the sperm tail. (b) After release of the plasma membrane with $0.04 \%$ Triton X100, WGA-FITC binding was not evident in the anterior acrosomal region and tail. Scale bars represent $10 \mu \mathrm{m}$.

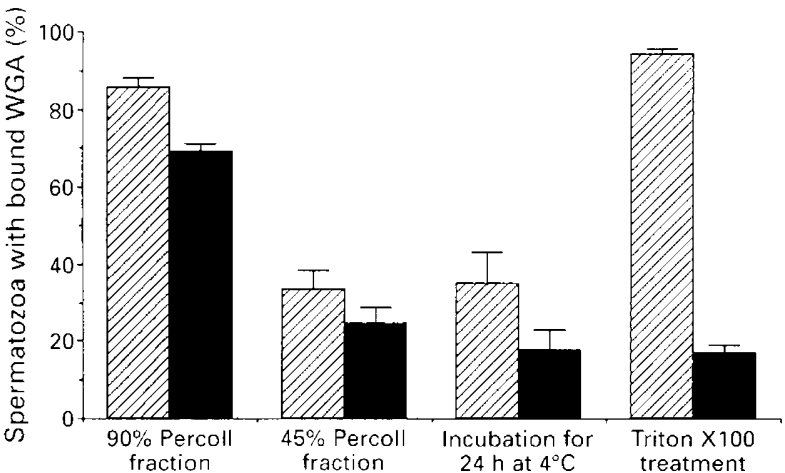

Fig. 4. Wheat germ agglutinin (WGA)-binding sites on the head of spermatozoa recovered in the $90 \%$ Percoll fractions after incubation for $24 \mathrm{~h}$ at $4^{\circ} \mathrm{C}$ and after treatment of spermatozoa with Triton X100. Comparison with untreated spermatozoa from $90 \%$ and $45 \%$ Percoll fractions. (2) WGA sites on sperm heads whatever the precise binding location; ( $)$ WGA sites at the anterior acrosomal region. Data are means \pm SEM from six experiments.

sWGA binding, whereas WGA could bind to more than $80 \%$ of spermatozoa from this fraction. Binding of WGA was positively related to sperm motility, whereas a negative relationship was observed for PNA, Con $A$ and sWGA binding.

PNA and Con A have been used to assess the acrosomal status of human spermatozoa (Mortimer et al., 1987; Holden et al., 1990). Mortimer et al. (1987) demonstrated specific PNA labelling of the outer acrosomal membrane, whereas Con A binds predominantly to the inner acrosomal membrane (Holden et al., 1990). In the study reported here, PNA, Con A and WGA (as sWGA) bound more than $80 \%$ of spermatozoa after release of the sperm plasma membrane with Triton X100. However, this treatment unmasked PNA- and Con A-binding sites at the anterior acrosomal region, i.e. on acrosomal

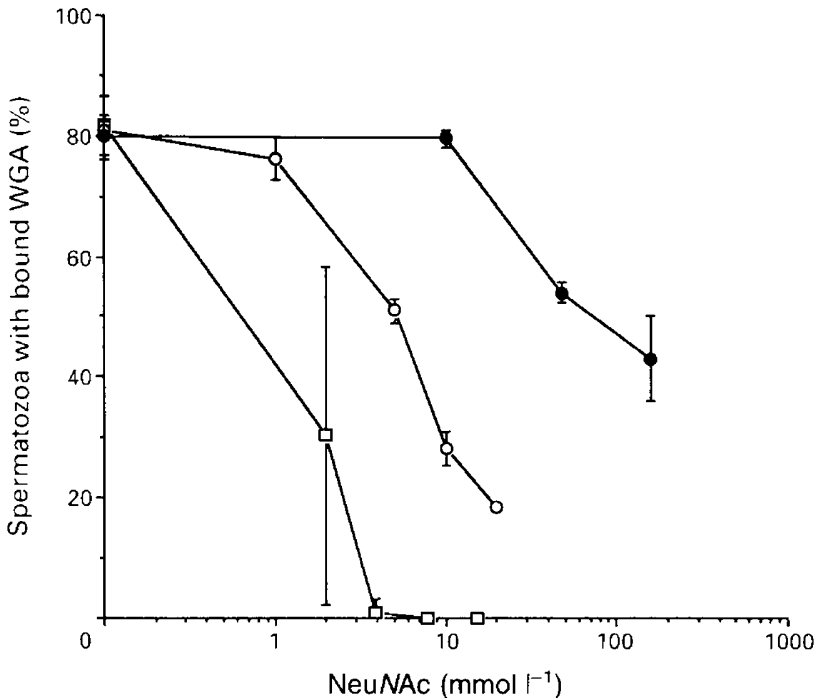

Fig. 5. Competitive inhibition of wheat germ agglutinin (WGA) binding on human sperm head with NeuNAc ( ) , fetuin () and bovine submaxillary mucin (-). WGA-fluorescein isothiocyanate (FITC) at $5 \mu \mathrm{g} \mathrm{ml}{ }^{-1}$ in $1 \mathrm{ml} \mathrm{B2}$ medium was incubated alone (control) or with increasing concentrations of either $N$-acetylneuraminic acid (NeuNAc), bovine submaxillary mucin (BSM) or bovine fetuin. After incubation for $\mathrm{I} \mathrm{h}$ at $37^{\circ} \mathrm{C}, 10^{\circ}$ spermatozoa $(100 \mu \mathrm{l})$ from the $90 \%$ Percoll fraction were added and incubated for a further $1 \mathrm{~h}$ at $37^{\circ} \mathrm{C}$. Spermatozoa were washed twice before observation under a microscope and the percentage of spermatozoa showing fluorescence on the head surface was estimated for each inhibitor tested. Concentrations of fetuin and BSM were expressed on the basis of NeuNAc content. Data are means \pm SEM from five (NeuNAc), two (fetuin) and six (BSM) experiments.

membranes of spermatozoa, whereas WGA binding sites on the anterior acrosomal region seemed to be released with the plasma membrane. After treatment of spermatozoa with Triton 


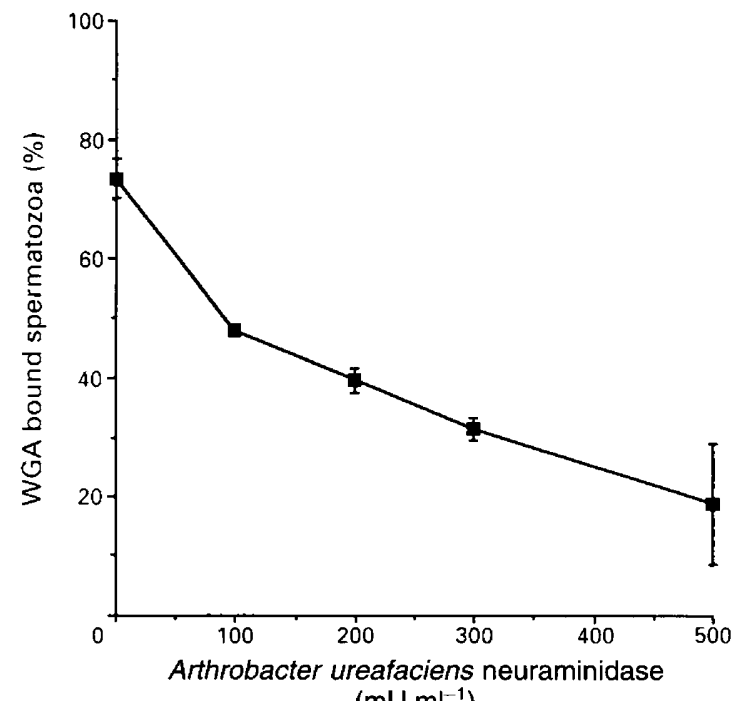

$\left(\mathrm{mU} \mathrm{ml}^{-1}\right)$

Fig. 6. Inhibition of wheat germ agglutinin (WGA) binding on the surface of human spermatozoa by neuraminidase. Spermatozoa were pretreated with increasing concentrations of neuraminidase from Arthrobacter ureafaciens before addition of WGA-fluorescein isothiocyanate (FITC) probes. The percentage of spermatozoa with bound WGA decreased for $200(P<0.001), 300(P<0.001)$ and 500 $(P<0.01) \mathrm{mU}$ neuraminidase $\mathrm{ml}^{-1}$ compared with the control. Each point represents the mean \pm SEM of two experiments.

Table 2. Average number of human spermatozoa bound per zona pellucida after sperm treatment with neuraminidase

\begin{tabular}{lcc}
\hline & \multicolumn{2}{c}{$\begin{array}{c}\text { Concentrations of Arthrobacter } \\
\text { ureafaciens neuraminidase }\end{array}$} \\
\cline { 2 - 3 } Donor & $0 \mathrm{mU} \mathrm{ml}^{-1}$ & $500 \mathrm{mU} \mathrm{ml}^{-1}$ \\
\hline 1 & $2.9 \pm 1.2(14)^{\mathrm{a}}$ & $18.0 \pm 2.1(15)^{\mathrm{a}}$ \\
2 & $3.4 \pm 0.5(13)^{\mathrm{b}}$ & $15.8 \pm 2.5(16)^{\mathrm{b}}$ \\
3 & $16.9 \pm 3.3(16)^{\mathrm{c}}$ & $35.4 \pm 5.3(16)^{\mathrm{c}}$ \\
\hline
\end{tabular}

Spermatozoa recovered from the $90 \%$ Percoll fraction were either untreated or treated for $1 \mathrm{~h}$ with $500 \mathrm{mU}$ Arthrobacter ureafaciens neuraminidase $\mathrm{ml}^{-1}$ at $37^{\circ} \mathrm{C}$ before labelling with fluorescein isothiocyanate stock solution. After washing by centrifugation, spermatozoa were resuspended in B2 medium and diluted to $5 \times 10^{5}$ spermatozoa $\mathrm{ml}^{-1}$. Five hundred microlitres of each sperm suspension was placed in macrowells of Nunclon delta dishes before addition of human zonae pellucidae. After $2 \mathrm{~h}$ incubation (at $37^{\circ} \mathrm{C}$ under $5 \% \mathrm{CO}_{2}$ ), zonae pellucidae were thoroughly rinsed in PBS-BSA then placed between slide and coverslip for observation under a microscope.

The number of human zonae pellucidae used is indicated in parentheses. ${ }^{\text {a.b }} p<0.001 ;{ }^{c} p<0.01$.

X100, WGA binding sites were observed particularly on the equatorial segment and the post-acrosomal region. This leads us to hypothesize that additional WGA binding sites could be unmasked on the equatorial segment and on posterior acrosomal regions after sperm plasma membrane release. In agreement with the work of Liu et al. (1991) on mouse spermatozoa, these results support the contention that WGAbinding sites are at the surface of intact human spermatozoa.

Although the exact mechanism of the capacitation process remains to be clarified, capacitation is viewed as a reversible phenomenon that results in a net decrease in negative surface charge, an efflux of membrane cholesterol, and an influx of calcium ions between the plasma and outer acrosomal membrane (reviewed by Langlais and Roberts, 1985). Holt (1980) suggested that sialic acid was responsible for the net negative surface charge of the spermatozoon, whereas the loss of the negative surface charge results from the removal of sialic acid (Srivastava and Farooqui, 1980). In the study reported here, we observed a marked decrease in binding to WGA, a lectin which recognizes sialic acid, after incubation for $24 \mathrm{~h}$ at $4^{\circ} \mathrm{C}$ compared with untreated sperm samples. Moreover, incubation of spermatozoa for $24 \mathrm{~h}$ at $4^{\circ} \mathrm{C}$ increased the acrosome reaction and fertilizing ability of human spermatozoa (Carrell et al., 1992). In the study reported here, the percentage of acrosome-reacted spermatozoa increased after incubation for $24 \mathrm{~h}$ at $4^{\circ} \mathrm{C}$. These results support the contention that there is a decrease of the net negative surface charge and an influx of calcium ions, two events characteristic of the sperm capacitation process. The percentage of spermatozoa with WGA bound anywhere on the sperm head and those with WGA bound on the anterior acrosomal region both decreased after incubation compared with that of untreated and Triton X100-treated spermatozoa. After incubation, WGA-binding sites on the plasma membrane were not evident, although the plasma membrane persisted. Similar results were obtained using sperm populations with low percentages of motile spermatozoa from the $45 \%$ Percoll fraction. However, sperm populations recovered from low Percoll density fractions present lower percentages of motile spermatozoa of normal morphology, and of mature spermatozoa compared with those from the $90 \%$ Percoll fractions (Le Lannou and Blanchard, 1988).

WGA and sWGA have been used to determine the number of cell surface receptors containing sialic acid (Monsigny et al., 1980). WGA can recognize both NeuNAc and GlcNAc, whereas sWGA recognizes only GlcNAc (Monsigny et al., 1980). The percentages of untreated spermatozoa with bound sWGA, PNA and Con A were similar $(<20 \%)$, whereas WGA bound more than $80 \%$ of untreated spermatozoa. However, sWGA and WGA bound high percentages of Triton X100treated spermatozoa without great differences between percentages of spermatozoa with lectins bound on the anterior acrosome. From these results, we cannot exclude the possibility that GICNAc is the only sugar residue recognized by both sWGA and WGA after demembranation with Triton X100. However, we found that NeuNAc was expressed on the plasma membrane of motile spermatozoa, especially at the anterior acrosomal region and that this expression was lost after capacitation or plasma membrane release or on spermatozoa (live or dead) that are immotile.

The sialic acid residue is generally located on the terminal position of carbohydrate chains (Schauer, 1985), usually in $\alpha(2,3)$ or $\alpha(2,6)$ linkage to galactose (Gal) or N-acetyl-Dgalactosamine (GalNAc) residues of glycoproteins or glycolipids. The secondary and tertiary structures of carbohydrate chains associated with the particular polysaccharide in the terminal position seem to play a very significant role in lectin binding, as shown by the much stronger interaction between lectins and native oligosaccharides than with the corresponding simple sugar. WGA requires the presence of terminal NeuNAC 

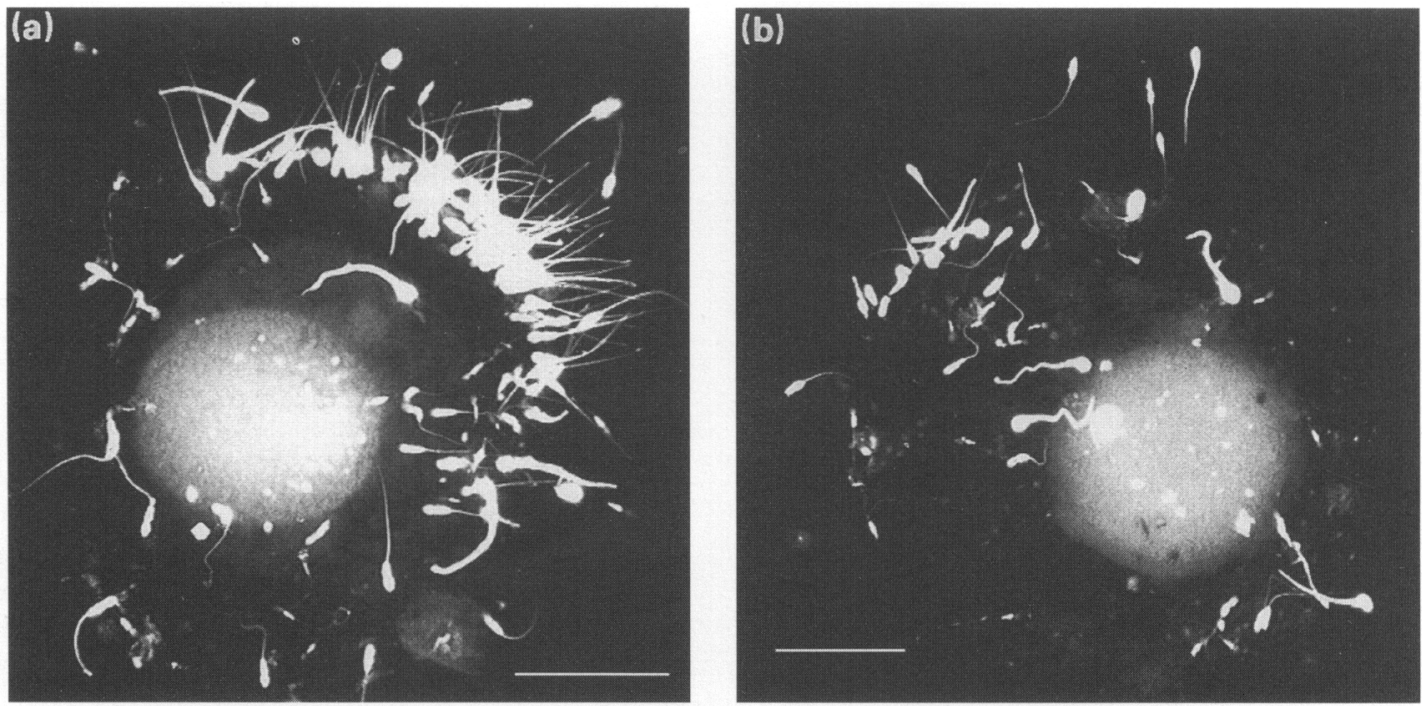

Fig. 7. Effect of pretreatment of spermatozoa with neuraminidase from Arthrobacter ureafaciens on human sperm-zona pellucida recognition. The ability of human spermatozoa to recognize human zona pellucida increased after sperm pretreatment with (a) $500 \mathrm{mU}$ Arthrobacter ureafaciens neuraminidase $\mathrm{mol}^{-1}$ compared with (b) control. Scale bars represent $50 \mu \mathrm{m}$.

in $\alpha(2,6)$ or $\alpha(2,3)$ linkage to the penultimate galactose or $N$-acetylgalactosamine for high-affinity binding (Monsigny et al., 1980; Furukawa et al., 1986) with a preference for $\alpha(2,6)$-linked NeuNAc (Wright, 1992). WGA was compared with three other lectins (SNA, MAL and LFA) with specificity for different NeuNAc-linkage to Gal/GalNAc to determine what kind of NeuNAc linkage was expressed on the plasma membrane of human spermatozoa. Only SNA binding showed a positive correlation with sperm motility as observed with WGA, but with a lower slope of regression curve indicating differences in specificity between these two lectins. SNA requires the presence of the disaccharide structure NeuNAc $\alpha(2,6) \mathrm{Gal} / \mathrm{GalNAc}$, whereas LFA and MAL require NeuNAc $\alpha(2,6)$ or $\alpha(2,3)$ GalNAc (Zeng and Gabius, 1992) or the trisaccharide structure NeuNAc $\alpha(2,3)$ Gal $\beta$ 1,4 GlcNAc/Glc (Wang and Cummings, 1988; Knibbs et al., 1991), respectively. At comparable NeuNAc concentrations, inhibition of WGA binding on spermatozoa was higher with bovine submaxillary mucin which contained $\alpha(2,6)$-linked NeuNAc than with fetuin containing a roughly equal proportion of $\alpha(2,3)$ - and $\alpha(2,6)$ linked NeuNAc (Heerze and Armstrong, 1990), NeuNAc $\alpha(2,3)$ lactose and free NeuNAc. The specificity of WGA for NeuNAc $\alpha(2,6) \mathrm{Gal} / \mathrm{GalNAc}$ glycoconjugate sequences was confirmed and the presence of such sequences on the plasma membrane of the human spermatozoa was demonstrated.

Capacitation is associated with the decrease in the net surface charge and loss of carbohydrates, including sialic acid, and such a decrease in electronegativity of the sperm surface occurs during treatment of spermatozoa with follicular fluid or neuraminidase (reviewed by Sidhu and Guraya, 1989). In the study reported here, treatment of spermatozoa with neuraminidase led to a dose-dependent decrease in WGA binding on the sperm surface and to the enhancement of the number of spermatozoa that could recognize the surface of the zona pellucida. This result differs from that of Lambert and Le (1984) in mouse (Mus musculus) in which treatment of capacited spermatozoa with high doses of neuraminidase (25000 mU neuraminidase $\mathrm{ml}^{-1}$ versus $500 \mathrm{mU} \mathrm{ml}^{-1}$ in this study) destroyed their ability to bind and fertilize eggs. However, this treatment had no effect on spermatozoa of Mus caroli. These observations are in agreement with the proposal that there are intra- and inter-species differences in molecular mechanisms involved in sperm-zona pellucida recognition.

Our results are consistent with an antigen-masking (antirecognition) function of sialic acid in several biological events and cells (reviewed in Takahashi, 1992). The release of sialic acid from many blood proteins by treatment with neuraminidase results in rapid clearance of these proteins from the blood circulation as a consequence of uptake by hepatic membrane receptors with specificity for galactose residues (Ashwell and Morell, 1975). Neuraminidase-treated and senescent erythrocytes which have binding sites with high specificity for $\beta$-galactose were sequestered by phagocytes (Kiehne and Schauer, 1992). We suggest that sialic acid protects the spermatozoon and sperm surface antigens against the immune system and that its absence from the sperm plasma membrane represents one of the capacitation events necessary for unmasking some sperm surface antigens implicated in zona pellucida recognition.

\section{References}

Ashwell G and Morell AG (1975) Studies on the hepatic recognition and removal of circulating glycoproteins. In Immune Recognition pp 531-532 Ed. AS Rosenthal. Academic Press, New York

Barthelemy C, Royere D, Hammahah S, Lebos C, Tharanne MJ and Lansac J (1990) Ultrastructural changes in membranes and acrosome of human sperm during cryopreservation Archives of Andrology 25 29-40

Bellvé AR and O'Brien DA (1983) The mammalian spermatozoon: structure and temporal assembly. In Mechanism and control of animal fertilization pp 55-137 Ed. JF Hartmann. Academic Press, New York 
Berger T, Marrs R and Moyer D (1985) Comparison of techniques for selection of motile spermatozoa Fertility and Sterility 40 268-273

Bolton VN and Braude PR (1984) Preparation of human spermatozoa for in vitro fertilization by isopycnic centrifugation on self generating density gradient Archives of Andrology 13 167-176

Brown CR, Von Glos KI and Jones R (1983) Changes in plasma membrane glycoproteins of rat spermatozoa during maturation in the epididymis Journal of Cell Biology 96 256-264

Carrell DT, Bradshaw WS, Jones KP, Middleton RG, Peterson CM and Urry RL (1992) An evaluation of various treatments to increase sperm penetration capacity for potential use in an in vitro fertilization program Fertility and Sterility 57 134-138

Eddy EM, Vernon RB, Muller $\mathrm{CH}_{r}$ Hahnel AC and Fenderson BA (1985) Immunodissection of sperm surface modifications during epididymal maturation American Journal of Anatomy 174 225-237

Furukawa K, Minor JE, Hegarty JD and Bhavanandan VP (1986) Interaction of sialoglycoproteins with wheat germ agglutinin-sepharose of varying ratio of lectin to sepharose Journal of Biological Chemistry 261 7755-7761

Giroux-Widemann V, Jouannet P, Pignot-Paintrand I and Feneux D (1991) Effects of $\mathrm{pH}$ on the reactivation of human spermatozoa demembranated with Triton X100 Molecular Reproduction and Development 29 157-162

Heerze LD and Armstrong GD (1990) Comparison of lectin-like activity of pertussis toxin with two plant lectins that have differential specificities for $\alpha(2-6)$ or (2-3)-linked sialic acid Biochemical and Biophysical Research Communications 172 1224-1229

Herrler G, Gross HJ, Imhof A, Brossmer R, Milks G and Paulson JC (1992) A synthetic sialic acid analogue is recognized by influenza $C$ virus as receptor determinant but is resistant to the receptor-destroying enzyme Journal of Biological Chemistry 26712 501-12 505

Holden CA, Hyne RV, Sathananthan AH and Trounson AO (1990) Assessment of the human sperm acrosome reaction using concanavalin A lectin Molecular Reproduction and Development 25 247-257

Holt WV (1980) Surface-bound sialic acid on ram and bull spermatozoa: Deposition during epididymal transit and stability during washing Biology of Reproduction $23847-857$

Hyne RV, Stojanoff A, Clarke GN, Lopata A and Johnston WIH (1983) Pregnancy from in vitro fertilization of human eggs after separation of motile spermatozoa by density gradient centrifugation Fertility and Sterility 45 93-96

Kiehne $K$ and Schauer R (1992) The influence of $\alpha$ - and $\beta$-galactose residues and sialic acid o-acetyl groups of rat erythrocytes on the interaction with peritoneal macrophages Biological Chemistry Hoppe-Seyler 373 1117-1123

Knibbs RN, Goldstein IJ, Ratcliffe RM and Shibuya N (1991) Characterization of the carbohydrate binding specificity of the leukoagglutinating lectin from Maackia amurensis Joumal of Biological Chemistry 266 83-88

Lambert $H$ and Le VA (1984) Possible involvement of a sialylated component of the sperm plasma membrane in sperm-zona interaction in the mouse Gamete Research 10 153-163

Langlais J and Roberts KD (1985) A molecular membrane model of sperm capacitation and the acrosome reaction of mammalian spermatozoa Gamete Research 12 183-224

Langlais J, Zollinger M, Plante L, Chapdelaine A, Bleau G and Roberts KD (1981) Localization of cholesteryl sulfate in human spermatozoa in support of hypothesis for the mechanism of capacitation Proceedings of the National Academy of Sciences USA 78 7266-7270

Lassalle B and Testart J (1991) Sequential transformations of human sperm nucleus in human egg Joumal of Reproduction and Fertility 91 393-402

Lassalle B and Testart J (1992) Relationship between fertilizing ability of frozen human spermatozoa and capacity for heparin binding and nuclear decondensation Joumal of Reproduction and Fertility 95 313-324

Le Lannou D and Blanchard Y (1988) Nuclear maturity and morphology of human spermatozoa selected by Percoll density gradient centrifugation or swim-up procedure Journal of Reproduction and Fertility 84 551-556

Liu DY, Lopata A, Johnston WIH and Baker HWG (1988) A human sperm-zona pellucida binding test using oocytes that failed to fertilize in vitro Fertility and Sterility 50 782-788

Liu HW, Wang JJ, Chao CF and Muller C (1991) Identification of two maturation-related, wheat-germ-lectin-binding proteins on the surface of mouse sperm Acta Anatomica 142 165-170

Magargee SF, Kunze E and Hammerstedt RH (1988) Changes in lectin-binding features of ram sperm surfaces associated with epididymal maturation and ejaculation Biology of Reproduction 38 667-685
Miller RL, Collawn JF and Fish WW (1982) Purification and macromolecular properties of a sialic acid-specific lectin from the slug Limax flavus Journal of Biological Chemistry 57 7574-7580

Monsigny M, Roche AC, Sene C, Magnet-Dana R and Delmotte F (1980) Sugar-lectin interactions: how does wheat-germ agglutinin bind sialoglycoconjugates? European Journal of Biochemistry 104 147-153

Mortimer D, Curtis EF and Miller RG (1987) Specific labelling by peanut agglutinin of the outer acrosomal membrane of the human spermatozoa Journal of Reproduction and Fertility 81 127-135

Orgebin-Crist MC and Fournier-Delpech S (1982) Sperm-egg interaction: evidence for maturation changes during epididymal transit Joumal of Andrology $3429-433$

Pandya IJ and Cohen J (1985) The leucocytic reaction of the human uterine cervix to spermatozoa Fertility and Sterility 43 417-421

Pereira MEA, Kabat EA, Lotan R and Sharon N (1976) Immunochemical studies on the specificity of the peanut (Arachis hypogaea) agglutinin Carbohydrate Research 51 107-118

Powell LD, Sgroi D, Sjoberg ER, Stamenkovic I and Varki A (1993) Natural ligands of the $\mathrm{B}$ cell adhesion molecule $\mathrm{CD} 22 \beta$ carry $N$-linked oligosaccharides with $\alpha-2,6$-linked sialic acids that are required for recognition Joumal of Biological Chemistry 268 7019-7027

Primakoff $\mathrm{P}$, Hyatt $\mathrm{H}$ and Tredick-Kline H (1987) Identification and purification of a sperm surface protein with a potential role in sperm-gg membrane fusion Journal of Cell Biology 104 141-149

Sapienza F, Verheyen G, Tournaye $H$, Janssens R, Pletincx I, Derde $M$ and Van Steirteghem A (1993) An auto-controlled study in in-vitro fertilization reveals the benefit of Percoll centrifugation to swim-up in the preparation of poor-quality semen Human Reproduction 8 1856-1862

Schauer R (1985) Sialic acids and their role as biological masks Trends in Biochemical Science 10 357-360

Shibuya N, Golstein IJ, Brookaert WF, Nsimba-Lubaki M, Peeters B and Peumans WJ (1987) The elderberry (Sambucus nigra L.) bark lectin recognizes the Neu5Ac( $\alpha 2-6) \mathrm{Gal} / \mathrm{GalNAc}$ sequence Joumal of Biological Chemistry 262 1596-1601

Sidhu KS and Guraya SS (1989) Cellular and molecular biology of capacitation and acrosome reaction in mammalian spermatozoa International Review of Cytology 118 231-280

Srivastava A and Olson GE (1991) Glycoprotein changes in the rat sperm plasma membrane during maturation in the epididymis Molecular Reproduction and Development 29 357-364

Srivastava PN and Farooqui AA (1980) Studies on neuraminidase activity of the rabbit endometrium Biology of Reproduction 22 858-863

Takahashi H (1992) The masking effect of sialic acid on Con A, PNA and SBA ectoderm binding sites during neurulation in the bantam chick embryo Anatomy and Embryology 185 389-400

Tomlinson MJ, White A, Barratt CLR, Bolton AE and Cooke ID (1992) The removal of morphologically abnormal sperm forms by phagocytes: a positive role for seminal leukocytes? Human Reproduction 7 517-522

Toowicharanont $\mathbf{P}$ and Chulavatnatol M (1983) Direct assay of bound sialic acids on rat spermatozoa from caput and cauda epididymis Journal of Reproduction and Fertility $67275-280$

Toshimori K, Araki S, Oura C and Eddy EM (199I) Loss of sperm surface sialic acid induces phagocytosis Archives of Andrology 27 79-86

Wang WC and Cummings RD (1988) The immobilized leukoagglutinin from seeds of Maackia amurensis with high affinity to complex-type Asn-linked oligosaccharides containing terminal sialic acid-linked $\alpha-2,3$ to penultimate galactose residues Journal of Biological Chemistry 263 4576-4585

Wright $\mathrm{CH}$ (1992) Crystal structure of a wheat germ agglutinin/glycophorin-sialoglycopeptide receptor complex Joumal of Biological Chemistry $26714 \quad 345-14352$

Yoshimatsu N, Yanagimachi R and Lopata A (1988) Zonae pellucidae of saltstored hamster and human eggs: their penetrability by homologous and heterologous spermatozoa Gamete Research 21 115-126

Yoshida M, Fuse G, Matsui T and Ouchi S (1992) Identification of sialic acids in cell adhesion molecule, contact site A from Dictyostelium discoideum Biochemical and Biophysical Research Communication 188 794-798

Zeng FY and Gabius HJ (1992) Sialic acid-binding proteins: characterization, biological function and application Zeitschrift für Naturforschung $47 \mathrm{c}$ $641-653$ 